\title{
Pancreatic Duct Obstruction in Rabbits Causes Digestive Zymogen and Lysosomal Enzyme Colocalization
}

\author{
Ashok Saluja, " Manju Saluja," Antonello Villa," Ubaldo Leli," Peter Rutledge," Jacopo Meldolesi, ${ }^{\star}$ and Michael Steer* \\ *Department of Surgery, Harvard Digestive Diseases Center, Beth Israel Hospital and Harvard Medical School, Boston, Massachusetts \\ 02215; and ${ }^{\ddagger}$ Department of Pharmacology, Consiglio Nazionale delle Richerche Center of Cytopharmacology \\ and Center for the Study of Peripheral Neuropathies, University of Milan, Italy 20229
}

\begin{abstract}
The pancreatic duct of anesthetized rabbits was cannulated and, in some animals, flow of pancreatic exocrine secretions was blocked by raising the cannula to a vertical position. Blockage for 3-7 $\mathrm{h}$ caused a rapid and significant rise in serum amylase activity and an increase in amylase activity within the pancreas. The concentration of lysosomal enzymes in the pancreas was not altered but they became redistributed among subcellular fractions and, as a result, an increased amount was recovered in the $1,000-g, 15-$ min pellet, which was enriched in zymogen granules. Immunofluorescence studies indicated that lysosomal enzymes become localized within organelles which, in size and distribution, resemble zymogen granules. They also contain digestive enzyme zymogens. Blockage of pancreatic secretions also caused lysosomal enzyme-containing organelles to become more fragile and subject to in vitro rupture. These changes noted after short-term pancreatic duct obstruction are remarkably similar to those previously noted to occur during the early stages of diet and secretagogue-induced experimental pancreatitis, observations that have suggested that colocalization of digestive enzyme zymogens and lysosomal hydrolases might result in intracellular digestive enzyme activation and be an important early event in the evolution of those forms of experimental acute pancreatitis.
\end{abstract}

\section{Introduction}

Considerable progress has been recently made in elucidating the cell biology of two experimental models of acute pancreatitis: one induced by feeding young female mice a choline-deficient, ethionine-supplemented diet and another induced by infusing rats with a dose of the secretagogue caerulein in excess of that which stimulates a maximal rate of pancreatic protein secretion. Those studies have suggested that intraacinar cell activation of digestive enzymes, resulting from their colocalization with lysosomal hydrolases such as cathepsin B, could be an important event in the ultimate development of cell injury (1-4). It is, however, not clear that these observations can be extrapolated to the situation in clinical pancreatitis, because the clinical disease obviously does not result from ethionine ingestion and is unlikely to be the result of supra-

Address reprint requests to Dr. Michael Steer, Department of Surgery, Beth Israel Hospital, 330 Brookline Avenue, Boston, MA 02215.

Received for publication 14 March 1988 and in revised form 7 June 1989.

J. Clin. Invest.

(C) The American Society for Clinical Investigation, Inc.

$0021-9738 / 89 / 10 / 1260 / 07 \$ 2.00$

Volume 84, October 1989, 1260-1266 maximal secretagogue stimulation. Rather, gallstone pancreatitis, which is the most common form of acute pancreatitis, appears to be triggered by passage of a stone into or through the terminal bile duct (5). It has been suggested that such a stone might obstruct the pancreatic duct, but the mechanism whereby pancreatic duct obstruction could result in pancreatitis has not been clarified.

In this communication, we report the results of studies that evaluated pancreatic acinar cell function shortly after the induction of pancreatic duct obstruction. They indicate that pancreatic duct obstruction leads to colocalization of digestive enzyme zymogens and lysosomal hydrolases within acinar cells.

\section{Methods}

CBZ-L-arginyl-arginine-B-naphthylamide was purchased from $\mathrm{Ba}$ chem, caerulein from Peninsula Laboratories, Inc. (Belmont, CA) $\left[{ }^{3} \mathrm{H}\right]$ phenylalanine $(15-25 \mathrm{Ci} / \mathrm{mmol})$ from Amersham Corp. (Arlington Heights, ID) and calf thymus DNA, B-naphthylamine, Triton $\mathrm{X}-100$, and secretin from Sigma Chemical Co. (St. Louis, MO). Antibodies and reagents used for immunolocalization were obtained from sources previously reported (4). The goat anti-rabbit rhodamine-labeled IgGs were from Cappel Laboratories (Malvern, PA). Ready-Solv was obtained from Beckman Instruments (Fullerton, CA) and all other reagents were of the highest available grade of purity.

Animal preparation. New Zealand white rabbits of either sex weighing $\sim 3 \mathrm{~kg}$ (Pine Acre Rabbitary, West Brattleboro, VT) were fasted overnight, lightly anesthetized, and a cannula (PE-50) introduced into the femoral vein. After midline laparatomy, an additional cannula (PE-10) was introduced into the pancreatic duct for a distance of $0.75 \mathrm{~cm}$ by extraduodenal puncture of the duct. The cannula was brought out through a stab wound and the laparotomy incision closed. Anesthesia was maintained throughout the experiment by the periodic intravenous administration of pentobarbital $(\sim 10 \mathrm{mg} / \mathrm{kg}$ per $\mathrm{h})$. Body temperature was monitored using an intrarectal probe and maintained using a heating pad. All animals were continuously infused with secretin $(8 \mathrm{CU} / \mathrm{kg}$ per h) to stimulate pancreatic juice production. Two groups of cannulated animals were studied: those with free-flowing secretions in which the drainage cannula was maintained in its original horizontal position and those with obstructed secretion in which, after a $1-h$ period of stabilization, the free end of the drainage cannula was lifted to a vertical position. In this latter group, pancreatic secretion equilibrated within the vertically positioned cannula at a level sufficient $(17-22 \mathrm{~cm})$ to exert a hydrostatic pressure that matched the secretory pressure of the pancreas. As indicated, some experiments also involved rabbits that were neither surgically manipulated in any way nor infused with secretin. That group served as an uninstrumented control group.

Protein synthesis. The in-vivo rate of pancreatic protein synthesis was evaluated by quantitating the incorporation of ${ }^{3} \mathrm{H}$ radioactivity into TCA-precipitable material after intravenous administration of $\left[{ }^{3} \mathrm{H}\right]$ phenylalanine, $(60 \mu \mathrm{Ci}$ /animal $)$ as previously described (6).

Subcellular fractionation. The excised, trimmed, and homogenized rabbit pancreas was separated into its various subcellular fractions 
using differential centrifugation. The protocol originally developed by Tartakoff and Jamieson (7) that we modified for studies in rat tissue (6) was again modified to permit optimum separation of rabbit pancreatic cell fractions. Briefly, pancreatic fragments were homogenized in cold $0.3 \mathrm{M}$ sucrose using three up-and-down strokes of a Dounce homogenizer. The resulting homogenate was centrifuged $\left(150 \mathrm{~g}, 10 \mathrm{~min}, 4^{\circ} \mathrm{C}\right)$ to pellet debris and unbroken cells, which were discarded. The supernatant after this low speed centrifugation was considered to contain $100 \%$ of each of the components measured. It was centrifuged $(1000 \mathrm{~g}$, $15 \mathrm{~min}, 4^{\circ} \mathrm{C}$ ) to obtain a zymogen granule-enriched pellet and supernatant. That supernatant was centrifuged $\left(12,000 \mathrm{~g}, 12 \mathrm{~min}, 4^{\circ} \mathrm{C}\right)$, yielding a lysosome and mitochondria-enriched pellet and a supernatant that was, in turn, centrifuged $\left(105,000 \mathrm{~g}, 60 \mathrm{~min}, 4^{\circ} \mathrm{C}\right)$ to obtain a microsome-enriched pellet and supernatant, which was considered to contain soluble elements. The various pellets obtained during fractionation were individually resuspended in $2 \mathrm{ml}$ of $0.3 \mathrm{M}$ cold $\left(4^{\circ} \mathrm{C}\right)$ sucrose.

Fragility of lysosomal enzyme-containing organelles. The supernatant obtained after low-speed centrifugation $\left(150 \mathrm{~g}, 10 \mathrm{~min}, 4^{\circ} \mathrm{C}\right)$ described above was subjected to centrifugation $(12,000 \mathrm{~g}, 12 \mathrm{~min}$, $4^{\circ} \mathrm{C}$ ) to obtain a combined zymogen granule-lysosome-mitochondria-enriched pellet as previously described (2). This pellet, arbitrarily considered to contain $100 \%$ of the lysosomal enzyme activity, was resuspended in $0.3 \mathrm{M}$ sucrose, and incubated for varying time intervals at $25^{\circ} \mathrm{C}$. The samples were then recentrifuged $\left(12,000 \mathrm{~g}, 12 \mathrm{~min}, 4^{\circ} \mathrm{C}\right)$ to separate the particulate from the soluble lysosomal enzyme activity, each of which was individually measured after separation of the pellet and supernatant.

Immunofluorescence studies. Pancreatic tissue fragments were fixed for $1 \mathrm{~h}$ with a mixture of ice-cold formaldehyde (4\%, freshly prepared from paraformaldehyde) and glutaraldehyde (0.25\%) in 125 $\mathrm{mM}$ phosphate buffer ( $\mathrm{pH} 7.3$ ), and then washed extensively in the buffer alone. The fixed samples were infiltrated with sucrose by immersion in solutions of increasing concentration (0.5-2.3 M), and finally frozen in freon 12 cooled with liquid nitrogen. Frozen samples were used for cutting $1-\mu \mathrm{m}$-thick cryosections in an Ultracut microtome equipped with an FC4 apparatus (Reichert Scientific Instruments, Vienna, Austria). Couples of consecutive cryosections were treated for $120 \mathrm{~min}$ at room temperature with either anti-zymogen or anti-cathepsin $D$ antisera (1:20 and 1:10, respectively) dissolved in a buffer containing 0.3 Triton X-100, $0.45 \mathrm{M} \mathrm{NaCl}$ and $20 \mathrm{mM}$ phosphate, pH 7.2. Analogous cryosections were treated with rabbit nonimmune IgG $(10 \mu \mathrm{g} / \mathrm{ml})$, and served as controls. After thorough washing, all the slices were incubated for $60 \mathrm{~min}$ in a solution of rhodamine-labeled goat anti-rabbit IgG antibodies $(20 \mu \mathrm{g} / \mathrm{ml})$ in $20 \mathrm{mM}$ phosphate buffer, $\mathrm{pH} 7.4$, supplemented with $450 \mathrm{mM} \mathrm{NaCl}$ and $0.3 \%$ Triton X-100. For additional details see reference 8. Fluorescent cryosections were examined using a photomicroscope (III; Carl Zeiss, Inc., Thornwood, NY). Pictures were taken at a magnification of 400 in the same areas of consecutive section couples, and the distribution of the two antigens was directly compared, as previously described (4), in the printed pictures.

Assays. Amylase activity was measured according to the method of Bernfeld (9), cathepsin B activity was measured fluorimetrically according to the method of McDonald and Ellis (10) as modified by Saluja et al. (2), and cathepsin D and B-galactosidase were measured according to the methods of Anson (11) and Furth and Robinson (12), respectively. DNA was measured fluorimetrically using the method of LaBarca and Paigen (13), whereas $N$-acetyl glucosaminidase was measured according to the method of Barrett and Heath (14).

Data presentation. The results reported in this communication represent observations made using at least three animals in each group for each experiment. Data are expressed as the mean \pm SEM for multiple determinations. Deviation bars in the figures indicate SEM values, while the absence of such bars indicates that the SEM value was too small to illustrate. Analysis of variance (Figs. 1, 2, and 5), regression analysis (Fig. 4), and the $t$ test were used to evaluate the significance of

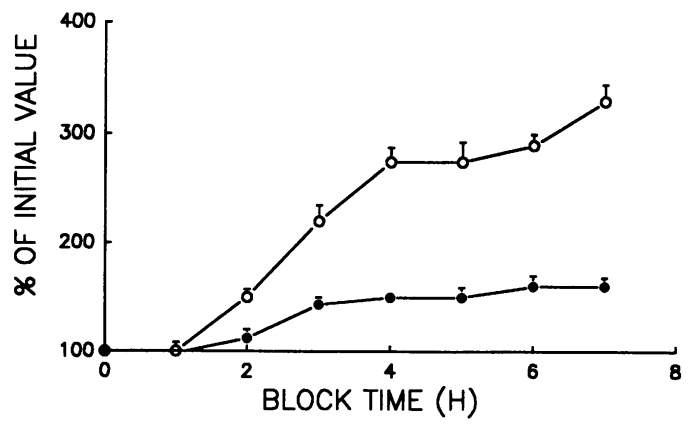

Figure 1. Effect of pancreatic duct obstruction on serum amylase. Serum amylase levels were measured at selected times after pancreatic duct cannulation and secretin infusion with (o) or without (๑) obstruction of pancreatic juice flow as described in the text.

observed changes, and a $P$ value of $<0.05$ was taken to indicate a significant difference.

\section{Results}

Serum amylase. The serum amylase activity was not altered within the first hour of ductal obstruction, but thereafter, ductal obstruction was associated with progressively rising serum amylase activity (Fig. 1). Cannulation of the pancreatic duct and stimulation with secretin caused the serum amylase to rise slightly even when the drainage cannula was not raised to a vertical position. We believe that this latter phenomenon indicates that, even in its horizontal position, the cannula represents a mild impediment to the free flow of secretions. The infusion of secretin, alone, in noncannulated control animals did not result in a demonstrable change in the serum amylase activity (data not shown).

Pancreatic protein synthesis and discharge. To evaluate the effects of ductal obstruction on pancreatic protein synthesis and discharge, animals were given a bolus of $\left[{ }^{3} \mathrm{H}\right]-$ phenylalanine $2 \mathrm{~h}$ after induction of ductal obstruction. The appearance and disappearance of ${ }^{3} \mathrm{H}$ radioactivity in TCA-precipitable material at varying times after pulse labeling is shown in Fig. 2. The time-dependent rate of increase and the maxi-

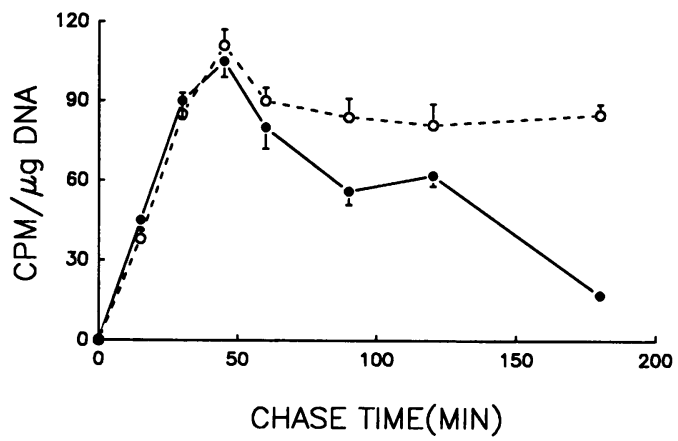

Figure 2. Appearance and disappearance of newly synthesized proteins in the pancreas. Newly synthesized proteins were pulse labeled with $\left[{ }^{3} \mathrm{H}\right]$ phenylalanine in cannulated animals $2 \mathrm{~h}$ after obstruction of pancreatic juice flow $(0)$ and in similar animals not subjected to obstruction (๑), as described in the text. At selected times, animals were killed and the TCA-precipitable radioactivity was measured in the pancreatic homogenates. 
Table I. Pancreatic Amylase Concentration

\begin{tabular}{lcc}
\hline \multicolumn{1}{c}{ Treatment } & $n$ & Amylase \\
\hline & & $U / m g$ DNA \\
Control & 3 & $0.65 \pm 0.10$ \\
Free-flowing secretion & 4 & $0.83 \pm 0.30$ \\
Blocked secretion & 10 & $1.40 \pm 0.20^{*}$ \\
\hline
\end{tabular}

Values are mean $\pm \mathrm{SE}$ of duplicate measurements in $n$ animals. Amylase activity was measured in the pancreatic homogenate as described in the text. The activity is expressed as milligrams of maltose released from soluble starch per minute per milligram of DNA. * Significantly different from that of control rabbits $(P<0.05)$.

mum extent of the incorporation of radioactivity into TCAprecipitable material was similar in animals with either freeflowing or obstructed ducts. This observation indicates that ductal obstruction for 2-3 h does not alter pancreatic protein synthesis. On the other hand, the time-dependent disappearance of TCA-precipitable radioactivity from the pancreas was altered by ductal obstruction. This disappearance of TCA-precipitable radioactivity results from the discharge of newly synthesized ${ }^{3} \mathrm{H}$-labeled proteins from the gland, a process that appears to be prevented by pancreatic ductal obstruction.

Pancreatic amylase concentration. The concentration of amylase was found to be increased in the pancreas after $3 \mathrm{~h}$ of ductal obstruction (Table I). This increase, presumably, results from continued digestive enzyme synthesis during the period in which discharge from the gland is reduced. A small but not significant increase in amylase concentration within the pancreas was also observed in cannulated animals not subjected to ductal obstruction.

Pancreatic concentration of lysosomal enzymes. The concentration of cathepsin B and $N$-acetyl glucosaminidase was measured in the pancreas $7 \mathrm{~h}$ after the onset of ductal obstruction and found to be not significantly different from either noncannulated control animals or cannulated animals with nonobstructed ducts (Table II). These observations indicate that ductal obstruction for $7 \mathrm{~h}$ does not alter the concentration of these two lysosomal hydrolases in the pancreas.

Subcellular distribution of lysosomal enzymes. The distribution of cathepsin B and of $N$-acetyl glucosaminidase among

Table II. Lysosomal Enzymes Concentration

\begin{tabular}{llc}
\hline & \multicolumn{2}{c}{ Lysosomal enzyme } \\
\cline { 2 - 3 } \multicolumn{1}{c}{ Treatment } & \multicolumn{1}{c}{ Cathepsin B } & N-Acetyl glucosaminidase \\
\hline Control & $36.6 \pm 4.0(3)$ & $315 \pm 25(3)$ \\
Free-flowing secretion & $33.7 \pm 6.3(4)$ & $353 \pm 33(3)$ \\
Blocked secretions & $39.0 \pm 3.5(10)$ & $374 \pm 41(3)$
\end{tabular}

Values represent means \pm SEM obtained for the number of animals indicated in parentheses. The enzyme activity was measured in the pancreatic homogenates as described in the text. Cathepsin B activity is expressed as nanomoles of B-naphthylamide formed per minute per milligram DNA. $N$-Acetyl glucosaminidase activity is expressed as micrograms of $p$-nitrophenol released per hour per milligram DNA.
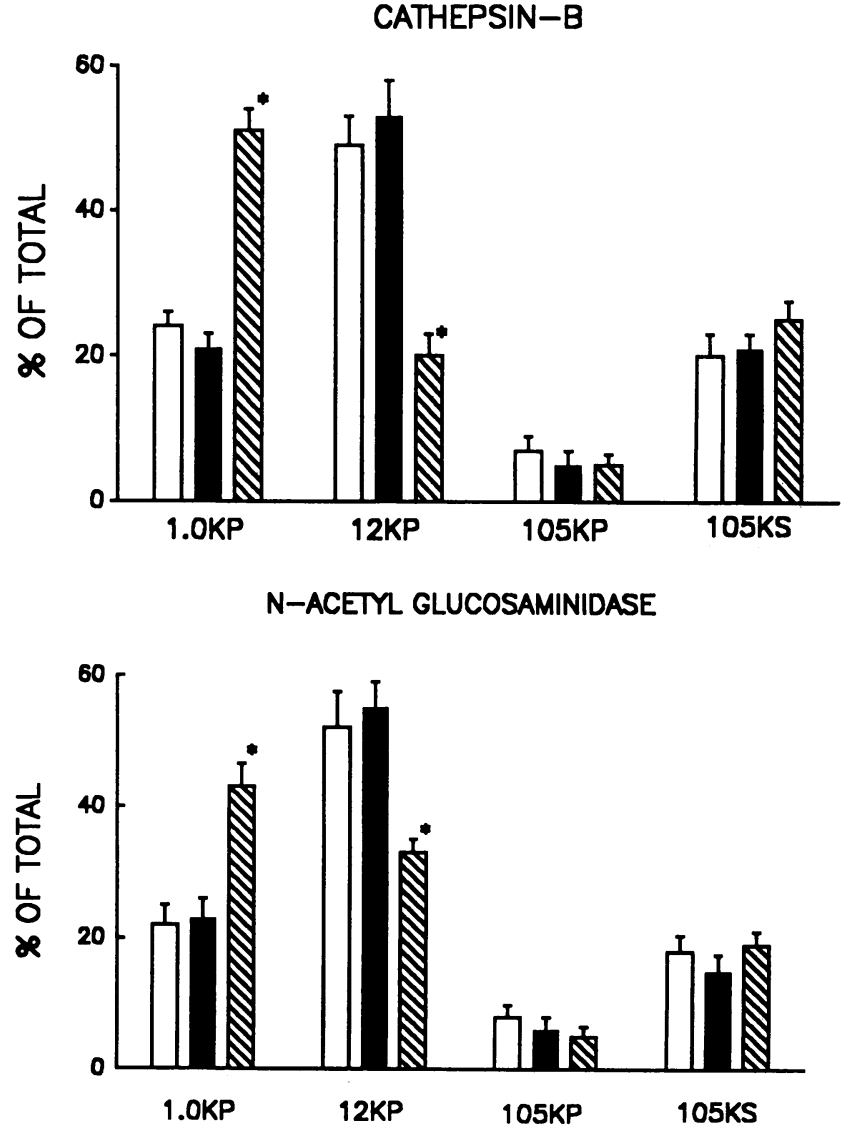

Figure 3. Subcellular distribution of lysosomal enzymes. Three groups of animals were studied, as follows: control (noninstrumented, open bars), free-flowing (cannulated but not obstructed, solid bars), and obstructed (cannulated and obstructed, hatched bars). The distribution of cathepsin B $(A)$ and of $N$-acetyl glucosaminidase $(B)$ among subcellular fractions prepared as described in the text was determined. The symbols are as follows: $1.0 \mathrm{KP}, 1000-\mathrm{g}, 10-$ min pellet; $12 \mathrm{KP}, 12,000-\mathrm{g}$, 12-min pellet; $105 \mathrm{KP}, 105,000-\mathrm{g}, 60-$ min pellet; $105 \mathrm{KS}, 105,000-\mathrm{g}$, 60 -min supernatant. Values are expressed as a percent of that measured in the 150-g, 10-min supernatant.

the various subcellular fractions is shown in Fig. 3. In control animals, as expected, most of the lysosomal enzyme activity $(\sim 50 \%)$ is recovered in the $12,000-\mathrm{g}, 12-\mathrm{min}$ pellet, whereas a smaller $(\sim 25 \%)$ yet significant portion is recovered in the heavier 1,000-g, 15-min pellet. This finding has been interpreted to mean that the pancreas contains a population of "heavy" lysosomes. Anesthesia, cannulation of the pancreatic duct, and infusion of secretin for $7 \mathrm{~h}$ did not result in a significant change in the distribution of cathepsin B or $\mathrm{N}$-acetyl glucosaminidase. In contrast, within $7 \mathrm{~h}$ of duct obstruction, most of the lysosomal enzyme activity $(\sim 55 \%)$ was recovered from the $1,000-\mathrm{g}, 15-\mathrm{min}$ fraction, whereas a corresponding smaller portion was found in the 12,000-g, 12-min pellet $(\sim 20 \%)$. Ductal obstruction was found to cause a similar redistribution of two other lysosomal hydrolases (cathepsin D and B-galactosidase) as well (data not shown). These observations indicate that pancreatic ductal obstruction causes lysosomal hydrolases to become redistributed and, as a result, localized in a fraction normally enriched in zymogen granules. The time-dependence 


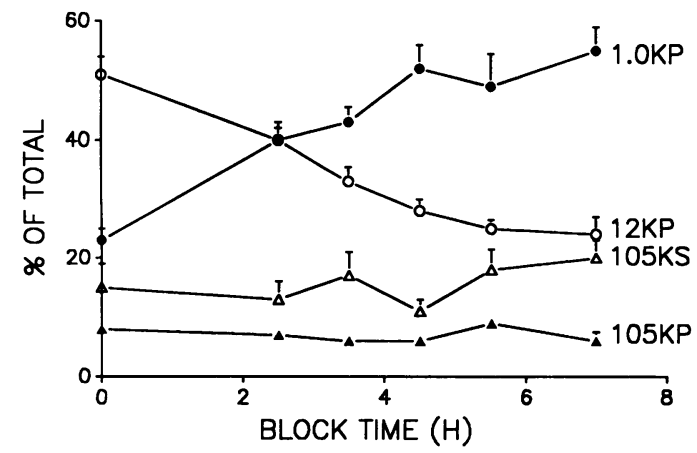

Figure 4. Time dependence of cathepsin B redistribution. Animals were killed at varying times after elevating the pancreatic duct cannula to create ductal obstruction. The distribution of cathepsin B among the various fractions was measured as described in the text. Other symbols are as in legend to Fig. 3. Values are expressed as a percent of the cathepsin B activity found in the 150-g, 10-min supernatant.

of this lysosomal enzyme redistribution is shown in Fig. 4. A significant shift in the localization of cathepsin B from the $12,000-\mathrm{g}, 12-\mathrm{min}$ to the $1,000-\mathrm{g}, 15-\mathrm{min}$ fraction was detected within $2.5 \mathrm{~h}$ of pancreatic duct obstruction. Shorter time periods were not evaluated.

Fragility of lysosomal enzyme-containing organelles. As shown in Fig. 5, incubation of samples obtained from cannulated but not obstructed animals at $25^{\circ} \mathrm{C}$ resulted in a slow but significant decrease in the particulate and increase in the soluble lysosomal enzyme activity. These changes reflect the known fragility of lysosomes. In contrast, a much more rapid and marked decrease in particulate and an increase in soluble lysosomal enzyme activity was noted during incubation of samples taken from duct-obstructed animals. Similar changes were noted when the activities of cathepsin D and B-galactosidase were measured (data not shown). These observations indicate that ductal obstruction increases the fragility of lysosomal enzyme-containing organelles.

Immunofluorescence studies. The intracellular distribution of zymogens and cathepsin $\mathrm{D}$ in sequential cryosections of control pancreatic acinar cells is illustrated in Figs. 6, $A$ and $B$. As can be seen, the zymogen fluorescence $(A)$, which was preferentially located toward the apex of acinar cells, was mostly related to discrete, spherical organelles. Based on their size and distribution, these organelles can be identified as zymogen granules. Cathepsin D immunofluorescence $(B)$ was either absent or very low (a few organelles distributed apparently at random within acinar cells). The images of Fig. 6, $A$ and $B$ closely resemble those previously observed when normal rat pancreas samples were examined (4) and are not markedly different from those noted when samples taken from cannulated but not obstructed rabbits (i.e., free-flowing) were examined (not shown).

Major changes in the intensity and distribution of fluorescence were noted when pancreas samples taken from rabbits with obstructed pancreatic ducts were examined (Fig. 6, $C-F$ ). The images obtained using antizymogen antibodies (Fig. 6, $C$ and $E$ ) resembled those for control samples, i.e., they revealed numerous fluorescence-positive organelles located near the cell apex. The number of these organelles, however, was mark-

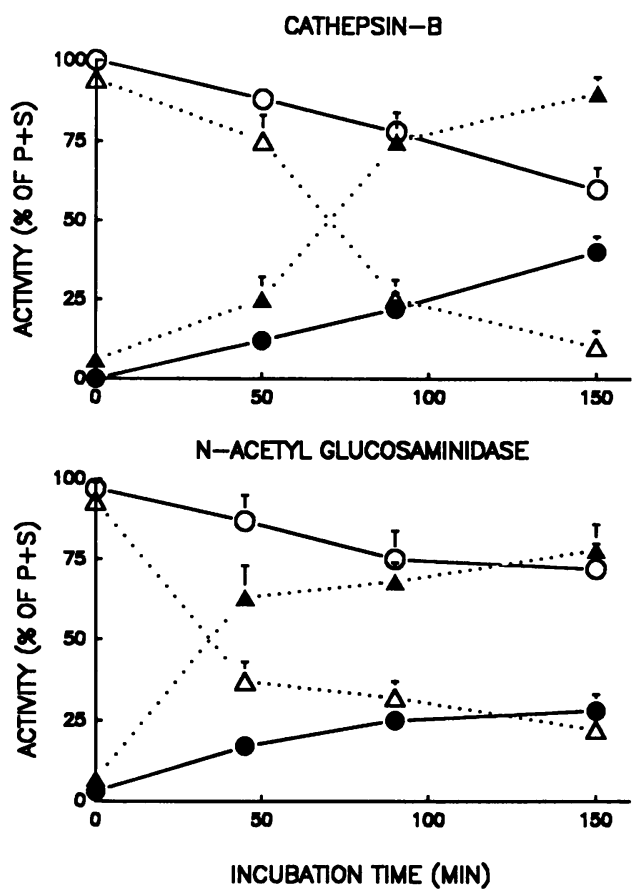

Figure 5. Fragility of lysosomal enzyme-containing organelles. $7 \mathrm{~h}$ after cannulation of the pancreatic duct with $(\Delta, \Delta)$ or without $(0, \bullet)$ ductal obstruction, animals were killed and the combined zymogen granule lysosome-enriched fractions were prepared as described in the text. These fractions were incubated at $25^{\circ} \mathrm{C}$ for varying times, after which particulate (open symbols) and soluble (closed symbols) cathepsin $\mathrm{B}(A)$ and $N$-acetyl glucosaminidase $(B)$ activities were measured. $P$ and $S$, particulate and soluble enzyme activity, respectively.

edly increased in the samples from duct-obstructed animals and the organelles appeared, therefore, to be much more closely packed than in the controls. In the cathepsin D-immunodecorated samples (Fig. 6, $D$ and $F$ ), the fluorescence changes caused by duct obstruction were even more marked. Most of the obstructed acinar cells exhibited a large population of fluorescence-positive organelles that were similar in size to zymogen granules and like zymogen granules, were located at the cell apex.

To achieve optimal and similar degree of resolution, the exposure time for cathepsin D samples was long (30 s), whereas that for zymogen samples was short $(6 \mathrm{~s})$. Consequently, the nonspecific fluorescence of the extracellular matrix appears much more prominent in the cathepsin $D$ images. This has been partially marked by the white broken lines in $C$ and $E$. To illustrate the actual relative intensity of the two immunolabelings, the inset in $C$ (which was taken from the area between the two solid vertical white lines in $C$ ) was photographically processed exactly as the cathepsin $\mathrm{D}$ images (i.e., 30-s exposure). Under these conditions, zymogen fluorescence was found to be much more intense than cathepsin D fluorescence.

Comparison of images obtained from sequential $1-\mu \mathrm{m}$ thick cryosections cut from the same block and decorated with either one of the antibodies allowed us to investigate whether, and to what extent, the two antigens were colocalized in the same organelles. As can be seen in the pairs $C$ and $D$ and $E$ and 

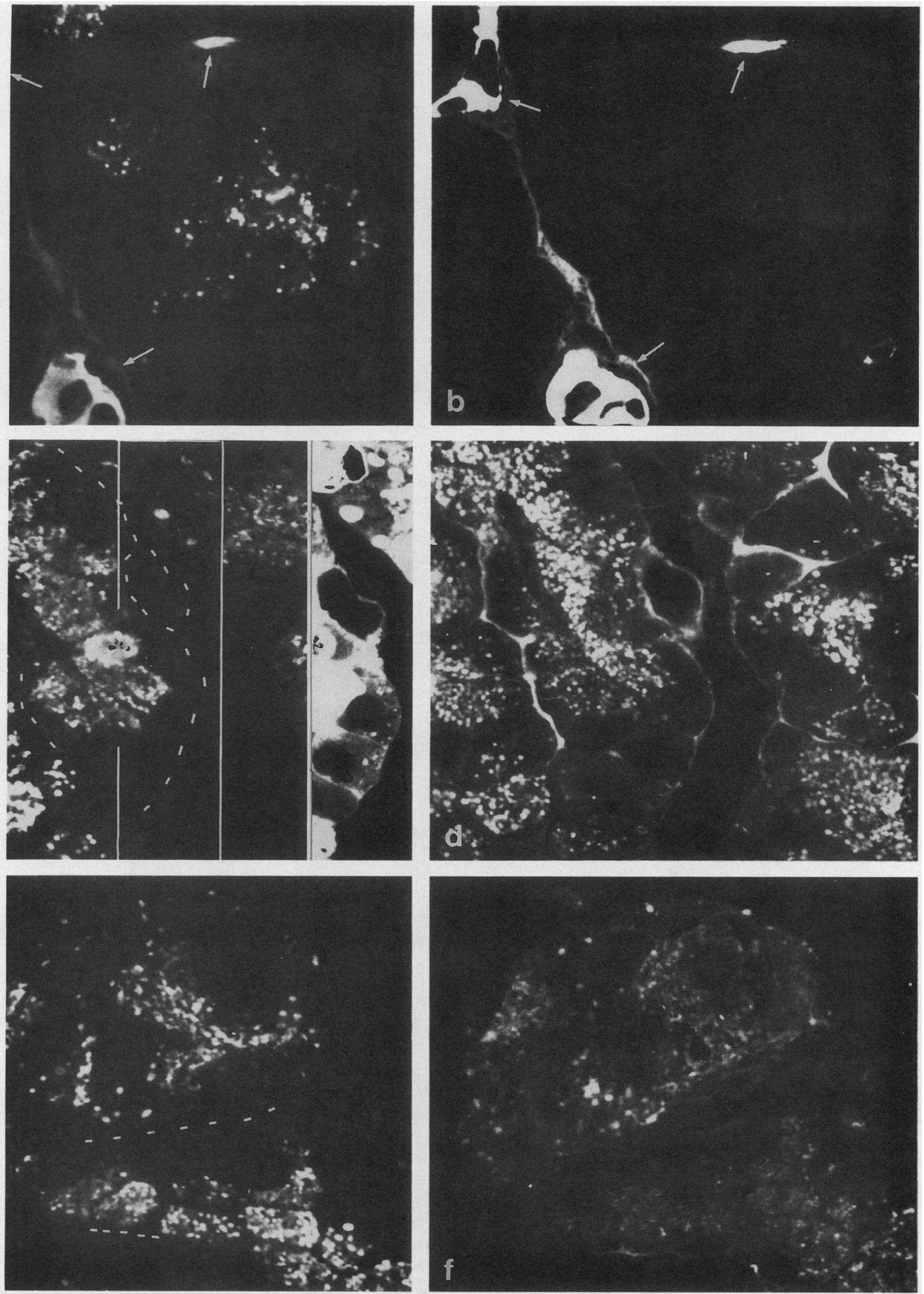

Figure 6. Antizymogen $(A, C$, and $E)$ and anticathepsin $\mathrm{D}(B, D$, and $F)$ immunofluorescence of successive 1- $\mu$ m-thick cryosections cut from the pancreas of control $(A$ and $B)$ and duct-obstructed $(C-F)$ rabbits. In control samples, antizymogen antibodies $(A)$ decorate a population of 
$F$, the two antigens have a very similar distribution. This similarity was noted for all acinar cells examined regardless of whether their degree of immunofluorescence was high $(C$ and $D)$ or low $(E$ and $F)$. In many individual organelles, some fluorescence could be observed with both of the antibodies in sequential sections. Frequently, strong fluorescence for one antigen was paralleled by only a weak signal for the other. As the diameter of the fluorescent organelles, which appear similar to zymogen granules, is most probably close to the thickness of the cryosections $(\sim 1 \mu \mathrm{m})$, the probability of uneven distribution of antigen in sequential sections is high and the nonparallel distribution of fluorescence intensity was to be expected. In addition, however, some organelles showed no correspondence whatsoever between zymogen and cathepsin $D$ labeling. This latter observation could indicate that detectable levels of zymogens and cathepsin D are not colocalized to all of the organelles or, alternatively, that the two sequential sections have dissimilar organelle distribution.

\section{Discussion}

In previous studies, we have extensively characterized the cell biology of two experimental forms of acute pancreatitis: diet induced and secretagogue induced $(2,3,15-18)$. Strikingly similar phenomena were noted to occur during the early stages of both of these models, although the ultimate degree of pancreatic injury differed considerably. Although these observations might be relevant to the genesis of those two experimental models of pancreatitis, their relevance to the clinical disease was not clear as clinical acute pancreatitis, a disease that is frequently associated with biliary tract stone disease, does not appear to involve either exposure to ethionine or excessive secretagogue stimulation. Rather, gallstone pancreatitis in humans appears to be precipitated by the passage of a stone through the terminal common bile duct (5).

The mechanism whereby such a stone might precipitate acute pancreatitis has been the subject of many studies and continues to be an issue of considerable controversy. Three hypotheses have been advanced, as follows: $(a)$ that the stone blocks drainage of bile and pancreatic juice into the duodenum but causes bile to reflux into the pancreatic duct; $(b)$ that stone passage through the sphincter of Oddi renders that structure incompetent and permits activated enzymes to reflux from the duodenum into the pancreatic duct, and $(c)$ that the stone or inflammation and edema resulting from the passage of the stone results in pancreatic duct obstruction, which, in a setting of continued pancreatic secretion, leads to ductal hypertension and rupture. There have been numerous objections to each of these hypotheses, not the least of which is that none of them clearly explains how activated digestive enzymes might gain access to the gland parenchyma.
The studies reported in this communication may provide an important missing link in the quest for an understanding of the events leading to pancreatitis. These studies clearly demonstrate that pancreatic ductal obstruction, under conditions in which physiological secretory pressures cannot be exceeded, has dramatic effects on pancreatic acinar cell function. Although protein synthesis continues, discharge of newly synthesized digestive enzyme proteins does not occur and the digestive enzyme concentration within the gland increases. There is a marked enlargement of the zymogen-containing organelle population located at the cell apex and those organelles are noted to contain lysosomal hydrolases. Subcellular fractionation experiments indicate that duct obstruction leads to a redistribution of lysosomal enzymes and that, as a result, lysosomal hydrolases become localized within a fraction that is enriched in digestive zymogens. Immunofluorescence studies indicate that lysosomal hydrolases are present within structures resembling zymogen granules that contain digestive enzyme zymogens. Together, these observations indicate that duct obstruction causes lysosomal hydrolases and digestive enzyme zymogens to become colocalized within the acinar cell. Because the lysosomal enzyme cathepsin B can activate trypsin $(19,20)$, this colocalization could result in digestive enzyme activation within acinar cells.

In our earlier studies $(3,4)$ involving diet- and secretagogue-induced pancreatitis, we concluded that the colocalization of lysosomal hydrolases and digestive zymogens observed in those models of pancreatitis was likely to be the result of crinophagy, i.e., discharge of secretory granules into lysosomes (21). Note, however, that in each of those models the colocalized lysosomal and digestive enzymes were found in cytoplasmic vacuoles, which were often much larger than the zymogen granule-sized organelles observed in this study. In contrast, no evidence of cytoplasmic vacuolization was noted after duct obstruction. Rather, the pattern of cathepsin D immunofluorescence was observed to change from being almost completely negative (most probably due to lack of antibody penetration into lysosomes) to being largely positive, with immunofluorescence localized in zymogen granule-sized organelles, which also contained digestive enzymes and were located near the cell apex. Although duct obstruction might stimulate crinophagy and thus lead to colocalization of digestive enzymes and lysosomal hydrolases by that mechanism, these observations suggest an alternative explanation. Duct obstruction, possibly by interfering with exocytosis, might interfere with the specific events which are involved in lysosomal enzyme transport from the Golgi complex to lysosomes (22), and thus cause lysosomal enzymes to be diverted into the regulated secretory pathway.

We have found that ductal obstruction causes lysosomal enzyme-containing organelles to become increasingly fragile. Similar observations have been made when diet- and secreta-

organelles (the zymogen granules) preferentially located at the cell apex, whereas the anticathepsin $\mathrm{D}$ pattern $(B)$ is negative. The elongated structures visible in both $A$ and $B$ are blood capillaries containing nonspecifically fluorescent erythrocytes (arrows). In the samples from ductobstructed animals $(C$ and $E$ ), zymogen-positive organelles appear similar in size and distribution to those seen in the controls but they are more numerous and packed in obstructed samples. Cathepsin D immunolabeling of obstructed samples $(D$ and $F)$ also reveals a population of fluorescence-positive organelles that have the same general distribution as the zymogen-positive organelles $(C$ and $E)$. Moreover, comparison of the two images in each couple ( $C$ and $D, E$ and $F$ ) reveals that numerous organelles are positive for both antigens. The inset in $C$ represents a longer exposure time ( $30 \mathrm{~s}$ ) for the segment enclosed within the two vertical solid white lines. Comparison of this inset with $D$ indicates that the intensity of fluorescence for zymogens is greater than that for cathepsin $\mathrm{D}$. The broken white lines in $C$ and $E$ describe the extracellular matrix at the base of acinar cells. $\times 1200$. 
gogue-induced models of pancreatitis have been studied. It is tempting to speculate that it is the colocalization of digestive zymogens with lysosomal hydrolases that leads to the observed increases in organellar fragility and to suggest that such increases in organellar fragility might result in the intracellular release of activated digestive enzymes.

Although the currently reported studies support the hypothesis that ductal obstruction may be important in the pathogenesis of gallstone pancreatitis, it is clear from these as well as many other studies that ductal obstruction alone is not sufficient to cause the morphological changes of pancreatitis. Clearly, other events must occur if the changes induced by ductal obstruction are to lead to pancreatic injury. Studies designed to identify those events may be of great importance because they are likely to be the ultimate determinants of the severity of pancreatitis and, even more important, because they may be subject to therapeutic alteration. The model of controlled pancreatic duct obstruction described here is likely to be of considerable value in future studies designed to address that issue.

\section{Acknowledgments}

The authors wish to thank Dr. F. M. Baccino (Department of General Pathology, University of Turin) for his kind gift of anticathepsin D antiserum.

This work was supported by National Institutes of Health grants 31396 and 31914.

\section{References}

1. Steer, M. L., and J. Meldolesi. 1987. The cell biology of experimental pancreatitis. N. Engl. J. Med. 316:144-150.

2. Saluja, A. K., S. Hashimoto, M. Saluja, R. E. Powers, J. Meldolesi, and M. L. Steer. 1987. Subcellular redistribution of lysosomal enzymes during caerulein-induced pancreatitis. Am. J. Physiol. 251:G508-G516.

3. Koike, H., M. L. Steer, and J. Meldolesi. 1982. Pancreatic effects of ethionine: blockade of exocytosis and appearance of crinophagy and autophagy precede cellular necrosis. Am. J. Physiol. 242 (Gastrointest. Liver Physiol. 5):G297-G307.

4. Watanabe, O., F. M. Baccino, M. L. Steer, and J. Meldolesi. 1984. Effects of supramaximal caerulein stimulation on the ultrastructure of rat pancreatic acinar cell: early morphological changes during the development of experimental pancreatitis. Am. J. Physiol. 246 (Gastrointest. Liver Physiol. 9):G457-G467.
5. Acosta, J. L., and C. L. Ledesma. 1974. Gallstone migration as a cause for acute pancreatitis. N. Engl. J. Med. 290:484-487.

6. Saluja, A., I. Saito, M. Saluja, M. J. Houlihan, R. Powers, J. Meldolesi, and M. L. Steer. 1985. In vivo rat pancreatic acinar cell function during supramaximal stimulation with caerulein. Am. J. Physiol. 249 (Gastrointest. Liver Physiol.):G702-G710.

7. Tartakoff, A., and J. E. Jamieson. 1974. Fractionation of guinea pig pancreas. Methods Enzymol. 31:41-59.

8. Hashimoto, S., B. Bruno, D. P. Lew, T. Pozzan, P. Volpe, and J. Meldolesi. 1988. Immunocytochemistry of calciosomes in liver and pancreas. J. Cell Biol. 107:2523-2531.

9. Bernfield, P. 1955. $\alpha$-Amylase. Methods Enzymol. 1:149-151.

10. McDonald, J. K., and S. Ellis. 1975. On the substrate specificity of cathepsin $B_{1}$ and $B_{2}$ including a new fluorogenic substrate for cathepsin $B_{1}$. Life Sci. 17:1269-1276.

11. Anson, M. L. 1936. The estimation of cathepsin with hemoglobin and the partial purification of cathepsin. J. Gen. Physiol. 20:565574.

12. Furth, A. J., and D. Robinson. 1965. Specificity and multiple forms of B-galactosidase in the rat. Biochem. J. 97:59-66.

13. Labarca, C., and K. Paigen. 1980. A simple, rapid and sensitive DNA assay procedure. Anal. Biochem. 102:344-352.

14. Barrett, A. J., and M. F. Heath. 1977. Lysosomal enzymes. In Lysosomal Enzymes, A Laboratory Handbook. 2nd ed. J. T. Dingle, editor. Elsevier/North-Holland Biomedical Press, Amsterdam. 19-145.

15. Lombardi, B., L. W. Estes, and D. S. Longnecker. 1975. Acute hemorrhagic pancreatitis (massive necrosis) with fat necrosis induced in mice by dl-ethionine fed with a choline deficient diet. Am. J. Pathol. 79:465-476.

16. Lampel, M., and H. Kern. 1977. Acute interstitial pancreatitis in the rat induced by excessive doses of a pancreatic secretagogue. Virchows Arch. A Pathol. Anat. Histol. 373:107-117.

17. Gilliland, L., and M. L. Steer. 1980. Effects of ethionine on digestive enzyme synthesis and discharge by mouse pancreas. Am. $J$. Physiol. 239 (Gastrointest. Liver Physiol. 2):G418-G426.

18. Saito, I., S. Hashimoto, A. Saluja, M. L. Steer, and J. Meldolesi. 1987. Intracellular transport of pancreatic zymogens during caerulein supramaximal stimulation. Am. J. Physiol. 253:G512-G521.

19. Greenbaum, L. A., and A. Hirshkowitz. 1961. Endogenous cathepsin activates trypsinogen in extracts of dog pancreas. Proc. Soc. Exp. Biol. Med. 107:74-76.

20. Steer, M. L., J. Meldolesi, and C. Figarella. 1984. Pancreatitis: the role of lysosomes. Dig. Dis. Sci. 19:934-938.

21. Farquhar, M. G. 1977. Secretion and crinophagy in prolactin cells. In Comparative Endocrinology of Prolactin. A. D. Dellman, J. A. Johnson, and D. M. Klachko, editors. Plenum Press, New York. 37-94.

22. Kornfeld, S. 1986. Trafficking of lysosomal enzymes in normal and disease states. J. Clin. Invest. 77:1-6. 